\title{
Combining Pest Control and Resistance Management: Synergy of Engineered Insects With Bt Crops
}

\author{
NINA ALPHEY, ${ }^{1,2,3}$ MICHAEL B. BONSALL, ${ }^{1}$ AND LUKE ALPHEY ${ }^{2,4}$
}

J. Econ. Entomol. 102(2): 717-732 (2009)

\begin{abstract}
Transgenic crops producing insecticidal toxins are widely used to control insect pests. Their benefits would be lost if resistance to the toxins became widespread in pest populations. The most widely used resistance management method is the high-dose/refuge strategy. This requires toxin-free host plants as refuges near insecticidal crops, and toxin doses intended to be sufficiently high to kill insects heterozygous for a resistant allele, thereby rendering resistance functionally recessive. We have previously shown by mathematical modeling that mass-release of harmless susceptible (toxin-sensitive) insects engineered with repressible female-specific lethality using release of insects carrying a dominant lethal ([RIDL] Oxitec Limited, United Kingdom) technology could substantially delay or reverse the spread of resistance and reduce refuge sizes. Here, we explore this proposal in depth, studying a wide range of scenarios, considering impacts on population dynamics as well as evolution of allele frequencies, comparing with releases of natural fertile susceptible insects, and examining the effect of seasonality. We investigate the outcome for pest control for which the plant-incorporated toxins are not necessarily at a high dose (i.e., they might not kill all homozygous susceptible and all heterozygous insects). We demonstrate that a RIDL-based approach could form an effective component of a resistance management strategy in a wide range of genetic and ecological circumstances. Because there are significant threshold effects for several variables, we expect that a margin of error would be advisable in setting release ratios and refuge sizes, especially as the frequency and properties of resistant alleles may be difficult to measure accurately in the field.
\end{abstract}

KEY WORDS resistance management, Bt crops, mathematical modeling, RIDL, high-dose/refuge strategy

Transgenic plants, mainly maize, Zea mays L., and cotton, Gossypium hirsutum L., producing insecticidal proteins derived from Bacillus thuringiensis (Berliner) (Bt) are widely used to control insect pests (James 2007). They can provide economic, health and environmental benefits (Shelton et al. 2002, Huang et al. 2003, Brookes and Barfoot 2006), so attention has focused on methods to prevent or reverse the spread of resistance to these toxins.

One approach is the high-dose/refuge strategy. This is a common tool for managing resistance to Bt crops and is mandatory in several countries. This aims to render any resistance functionally recessive by using such high toxin concentrations that heterozygotes do not survive. Refuges of host plants that produce no toxins (non-Bt), provide a source of susceptible genotypes to dilute the frequency of resistant $(r)$ alleles. Theoretical models, now supported by empirical ev-

\footnotetext{
${ }^{1}$ Mathematical Ecology Research Group, Department of Zoology, University of Oxford, South Parks Rd., Oxford OX1 3PS, United Kingdom.

${ }^{2}$ Oxitec Limited, 71 Milton Park, Oxford OX14 4RX, United Kingdom.

${ }^{3}$ Corresponding author, e-mail: luke.alphey@oxitec.com.

${ }^{4}$ Department of Zoology, University of Oxford, South Parks Rd., Oxford OX1 3PS, United Kingdom.
}

idence from monitoring, predict that this strategy slows or prevents the spread of resistance (Alstad and Andow 1995; Gould 1998; Carrière and Tabashnik 2001; Tabashnik et al. 2005, 2008).

In principle, instead of refuges an alternative source of susceptible $(s)$ alleles could be provided by massrearing susceptible insects and releasing them into the environment. In the sterile insect technique (SIT), a control method used to eliminate or suppress pest populations, insects are mass-reared, sterilized by irradiation, and released (Dyck et al. 2005); however, no viable hybrids are formed so there is no introgression of alleles into the wild population. Releasing natural, fertile, susceptible insects would allow such introgression, but released fertile females would contribute to population growth (very few reliable sex-separation methods have been developed for large-scale use in SIT, so generally both sexes would be released). Genetic technology (release of insects carrying a dominant lethal [RIDL], Oxitec Limited, United Kingdom) could modify the SIT by releasing insects that are not irradiated but instead are homozygous for one or more dominant lethal genetic constructs that are repressible during mass-rearing (Thomas et al. 2000, Alphey 2007, Alphey et al. 2007a). A female-lethal version of RIDL, with male insects homozygous for 
one or more female-specific dominant lethals ("RIDL males"), has been constructed in several species, including the major pests Mediterranean fruit fly, Ceratitis capitata (Wiedemann) (Fu et al. 2007), and Mexican fruit fly, Anastrepha ludens (Loew) (K. C. Stainton et al., unpublished data). $\mathrm{F}_{1}$ progeny of RIDL males and wild females inherit a dominant femalespecific lethal; the $\mathrm{F}_{1}$ females die, thereby reducing the reproductive potential of the wild population, but the $F_{1}$ males are viable and fertile, so alleles from the mass-reared strain might be introgressed into the wild population. We previously proposed that control programs could combine the release of such engineered insects with plants incorporating Bt or similar toxins to manage pests with fully recessive resistance where the toxins are fully lethal to susceptible genotypes (Alphey et al. 2007b). Here, we investigate applying this approach to a wider range of ecological and genetic circumstances.

The evolution of allele frequencies depends on selection pressure due to relative areas of Bt crop and refuge, and relative fitnesses of the genotypes in each. There are critical threshold proportions for habitat division that determine equilibrium allele frequencies (Alphey et al. 2008). If susceptible (ss) homozygotes are released each generation at a constant ratio to the wild population, these thresholds increase, allowing for equivalent resistance management to be provided by a smaller refuge (Alphey et al. 2007b), which could increase crop yields.

Our previous work, in which we explored fully recessive resistance (where the $r$ allele is recessive on $\mathrm{Bt}$ and non-Bt plants so that both the effects and fitness costs are recessive), found that release of RIDL males slows the spread of resistance and sufficient releases can prevent or reverse the spread of the $r$ allele (Alphey et al. 2007b). This analysis suggested that release of RIDL male insects, at release ratios considerably lower than those typical for effective SIT pest suppression programs, could allow a significant reduction in refuge size while maintaining equivalent resistance management benefits.

There is a tension between the benefit of using refuges to delay or prevent the spread of resistance and the benefit foregone by not planting insecticidal plants there (which would reduce crop damage by killing pest insects). In contrast, RIDL release could contribute simultaneously to reducing pest densities and managing resistance. The presence of released males will lead to some females mating with those instead of with wild males, so assuming that reproduction was not limited by the availability of males, release of males only does not increase the number of progeny (the cause of crop damage). The femalelethal effect of the RIDL construct would therefore be expected to have a population suppression effect synergistic with that provided by the Bt crops. Bt crops greatly reduce population sizes of susceptible insects (Caprio 2001, Carrière et al. 2003, Sisterson et al. 2004); the additional suppression provided by the RIDL strategy should improve this and might in some cases result in local elimination of the pest. To explore this potential synergy, which would cause desirable effects on population size as well as on resistant allele frequency, we must combine a population dynamics approach within the population genetics framework.

Whatever the approach used, resistance management analyses typically focus on preventing the spread of resistance from a very low initial level. A RIDL release program potentially could also be deployed as remedial action where resistance has become widespread. Higher release ratios, more typical of SIT population suppression programs, could achieve a much more rapid reduction in $r$ allele frequency than would be possible using only refuges (even with no Bt crops, i.e., $100 \%$ refuge, the rate of recovery would otherwise be driven only by the fitness cost of resistant insects on non-Bt plants).

As well as a low initial $r$ allele frequency, it is common for modeling studies to assume that Bt crops kill all susceptible larvae and that resistance is recessive. These assumptions are consistent with available evidence for some pests but not for others; there is a need also to understand the evolution of resistance in pests for which the toxins are not high-dose. For example, CrylAc cotton does not kill all susceptible larvae of the economically important pests bollworm, Helicoverpa zea (Boddie), and Helicoverpa armigera (Hübner), and some laboratory-selected resistance is not recessive (EPA 1998, Gould 1998, Akhurst et al. 2003, Gujar et al. 2007, Luttrell and Ali 2007). It is often also assumed that fitness costs exist and are recessive. However, fitness costs observed in resistant strains range from undetectable to substantial, and recessive to partially dominant, and may be environment-dependent (Ramachandran et al. 1998, Tang et al. 1999, Liu et al. 2001, Bates et al. 2005, Carrière et al. 2005a, Raymond et al. 2005).

The resistance trait in some species has been shown to have further fitness costs for overwintering (in affected regions), observed as reduced emergence from diapause (Alyokhin and Ferro 1999, Carrière et al. 2001b, Bird and Akhurst 2004). Conversely, continuous crop cultivation throughout the year is thought to be one key factor responsible for significant damage to CrylF Bt corn (maize) by larvae of fall army worm Spodoptera frugiperda (J.E. Smith) in Puerto Rico in 2006 (Reynolds 2007). We investigate the impact of seasonality on our results and conclusions.

Here, through mathematical modeling, we explore these issues for the proposed RIDL strategy for resistance management. We examine the relationship between critical release ratio, initial allele frequency and equilibrium allele frequency, first considering the evolution of resistant allele frequency for recessive resistance with nonrecessive fitness costs. We then explore the interaction between pest control and resistance management by modeling relative population size over time (and compare this with release of wild type, fertile, susceptible insects instead of RIDL males), and then consider the effect of seasonality on our conclusions about fully recessive resistance. Finally we investigate scenarios where $\mathrm{Bt}$ crops are not high dose 
and either resistance or fitness costs vary in dominance.

\section{Materials and Methods}

Underlying Population Genetics Model. We used a deterministic, discrete-generation population genetic model (Carrière and Tabashnik 2001, Alphey et al. 2008) and assume a closed homogeneous population, with random mating, and no immigration, emigration or mutation. We assume a 1:1 sex ratio. Larvae, the Bt-susceptible life stage, are assumed to spend their whole development either on Bt crops or in the refuge; dispersal occurs after emergence as adults and before mating.

We assume a fixed crop population, a proportion $\Phi$ of which are Bt plants, so the (non-Bt) refuge size is $1-\Phi$. Hard selection (Wallace 1968) acts in these two environments, with the number of larvae surviving to reproductive maturity from each environment depending on the relative fitness values of the eggs laid there. Larval susceptibility to Bt toxins is assumed to be controlled by a single autosomal locus with two alternative alleles: resistant $r$ (frequency $p$ ) and susceptible $s$ (frequency $q, p+q=1$ ). There are three possible genotypes at this locus: $s s, s r$, and $r r$.

All insects are affected by Bt toxins. We assume that all fitness costs associated with the $s$ and $r$ alleles take effect during the larval stage. Assuming that larvae are distributed at random across $\mathrm{Bt}$ and non-Bt crops, the average relative fitness of larvae of genotype $i(i: s s, s r$, or $r r$ ) across the area is

$$
\Omega_{i}=\omega_{i} \Phi+v_{i}(1-\Phi)
$$

where $\omega_{i}$ is the relative fitness of larvae on Bt crops and $v_{i}$ is relative fitness of larvae in non-Bt crops. Fitness is measured relative to $s s$ homozygotes on non- $\mathrm{Bt}$ plants (so $v_{s s}=1$ ). By definition of resistance $\omega_{r r}>\omega_{s s}$ We assume that no genotype is fitter on Bt crops than on non-Bt plants $\left(v_{i} \geq \omega_{i}\right)$. We assume that the resistant allele may have a fitness cost (on non-Bt plants $v_{r r} \leq v_{s r} \leq v_{s s}=1$ ), and that on $\mathrm{Bt}$ crops resistance is not over- or underdominant $\left(\omega_{s s} \leq \omega_{s r} \leq \omega_{r r}\right)$. Resistance may be incomplete $\left(\omega_{r r} \leq v_{r r}\right)$.

With no release of insects, the change in $r$ allele frequency is given by

$$
\Delta p=\frac{p q\left(p \Omega_{r r}+(q-p) \Omega_{s r}-q \Omega_{s s}\right)}{q^{2} \Omega_{s s}+2 p q \Omega_{s r}+p^{2} \Omega_{r r}}
$$

Using equation 2 , we have demonstrated that there are two critical threshold values, $\Phi_{1}$ and $\Phi_{2}$, resulting from the interplay between the costs and benefits of resistance, that determine the possible outcomes for the $r$ allele (Alphey et al. 2008). The outcome is determined by the proportion of $\mathrm{Bt}$ crops $(\Phi)$ in relation to these thresholds and, in some cases, by the allele's initial frequency $\left(p_{0}\right)$.

$$
\Phi_{1}=\frac{\left(1-v_{s r}\right)}{\left(1-v_{s r}+\omega_{s r}-\omega_{s s}\right)}
$$

$$
\Phi_{2}=\frac{\left(v_{r r}-v_{s r}\right)}{\left(v_{r r}-v_{s r}+\omega_{s r}-\omega_{r r}\right)}
$$

where $\Phi_{1}<\Phi_{2}$, if the proportion of Bt crops is below or equal to $\Phi_{1}$ the resistant allele will decline to extinction; if $\Phi$ is above or equal to $\Phi_{2}$, the resistant allele will go to fixation; if $\Phi$ lies between $\Phi_{1}$ and $\Phi_{2}$, the frequency of the resistant allele will settle at a stable internal equilibrium value (i.e., between 0 and 1 ) that can be calculated.

Where $\Phi_{1}>\Phi_{2}$, the resistant allele will go either to extinction or fixation, depending on whether the proportion of Bt crops is below or above a third threshold $\Phi_{3}$. The value of $\Phi_{3}$ is dependent on the initial frequency of the resistant allele $p_{0} . \Phi_{3}$ lies between $\Phi_{1}$ (the two are equal when $p_{0}=0$ ) and $\Phi_{2}$ (they are equal when $p_{0}=1$ ), and decreases as $p_{0}$ increases.

In special cases, where the $r$ allele is recessive on both Bt and non-Bt plants, dominant on both or codominant on both, one of equations 3 or 4 is not defined or they are equal, and there is a single threshold determining whether the $r$ allele goes to fixation or extinction.

To explore resistance with a range of genetic parameter values, it is helpful to define the dominance of resistance and the dominance of the fitness costs of resistance. We then vary the relative fitness values by changing those parameters. We define the dominance of resistance $h(0 \leq h \leq 1)$ as (Tabashnik et al. 2008)

$$
h=\frac{\omega_{s r}-\omega_{s s}}{\omega_{r r}-\omega_{s s}}
$$

(so $h=0$ for recessive resistance and $h=1$ for dominant resistance) and, where appropriate, calculate heterozygote relative fitness on Bt plants using $h$

$$
\omega_{s r}=(1-h) \omega_{s s}+h \omega_{r r}
$$

Similarly, we define the dominance of the fitness costs of resistance $g(0 \leq g \leq 1, g=0$ for recessive costs and $g=1$ for dominant costs) as

$$
g=\frac{v_{s r}-v_{s s}}{v_{r r}-v_{s s}}=\frac{1-v_{s r}}{1-v_{r r}}
$$

so that heterozygote relative fitness in the refuge becomes

$$
v_{s r}=(1-g) v_{s s}+g v_{r r}=1-g\left(1-v_{r r}\right)
$$

Release of Wild-Type (WT) Insects. To extend this basic model to incorporate the effects of the release of fertile wild-type insects, we assume that at each generation, as adults emerge before mating, mass-reared adult insects are released at a fixed ratio (the "release ratio") to the number of males in the wild population in that generation that survived to reproductive maturity. All released adults are assumed to be homozygous susceptible to Bt toxins (ss). Males and females are released in equal numbers. Wild and released adults mix homogeneously and mate at random.

With release of homozygote susceptible adults (ss wild type) in ratio $d: 1$ to naturally emerging males in the wild, the change in $r$ allele frequency is given by 


$$
\Delta p=\frac{p\left(p q \Omega_{r r}+\left(q+\frac{1}{2} d\right)(q-p) \Omega_{s r}-\left(q+\frac{1}{2} d\right)^{2} \Omega_{s s}\right)}{\left(q+\frac{1}{2} d\right)^{2} \Omega_{s s}+2 p\left(q+\frac{1}{2} d\right) \Omega_{s r}+p^{2} \Omega_{r r}}
$$

In practice, rather than determining the $r$ allele frequency by calculating $\Delta p$ from equation 9 , we used a simulation approach that involved calculating the frequencies of every genotype (Alphey et al. 2007b). This approach allowed us to analyze the model in the same way for both release of wild-type insects and the more complicated case of release of engineered insects.

Release of Engineered Insects. We further extend the model to reflect the release of males carrying a dominant female-lethal RIDL construct, which renders female progeny inviable but allows male progeny to survive. For this purpose, we assume that at each generation, as adults emerge before mating, massreared adult RIDL males are released at a fixed ratio (the "RIDL release ratio") to the number of males in the wild population (of any genotype) that survived to reproductive maturity. Females mate with wild or released males at random.

All engineered released males are assumed to be homozygous susceptible to $\mathrm{Bt}$ toxins (ss), and homozygous for the RIDL construct. There are two possible alleles at the insertion site of the RIDL construct: $L$, representing insertion of the construct, and the wild-type allele $w$, representing absence of the construct. We assume the RIDL insertion and the locus controlling resistance to $\mathrm{Bt}$ are not linked and neither of these is sex-linked.

We assume the RIDL construct imposes a sex-specific fitness cost $\varepsilon$ during the larval stage. The average relative fitness of larvae of genotype $i$ is then

$$
\Omega_{i}=\left(1-\varepsilon_{i}\right)\left[\omega_{i} \Phi+v_{i}(1-\Phi)\right]
$$

where $\varepsilon_{i}$ is the sex-specific, relative fitness cost imposed by RIDL.

This results in a complex system of 18 difference equations that cannot be reduced to expressions in terms of $p$. There are no analytical solutions for allele or genotype frequencies or for the critical release ratios or critical refuge sizes.

Population Dynamics. $R_{0}$ is the average number of progeny produced per adult pest insect that survive to adulthood in a density-independent population, commonly expressed as female offspring per adult female. For agricultural pests under field conditions, after taking into account factors such as egg hatch rates, predation and pathogens that are common to all genotypes throughout the habitat, $R_{0}$ might typically range from five tol0 but can be much higher (Dyck et al. 2005). The population size is determined by

$$
N_{t+1}=2 R_{0} F_{t} \sigma_{t}
$$

where $N_{t}$ is the population size of mature adults at generation $t$ relative to the initial pest density (set $N_{0}=1$ ), of which $F_{t}$ are female. The growth rate is $2 R_{0}$ (each adult female contributes $R_{0}$ male and $R_{0}$ female larvae to the next generation) and $\sigma_{t}$ is the proportion of offspring surviving to maturity (simulated by applying the fitness costs of $r$ alleles, Bt toxins and the RIDL construct as appropriate to each genotype). $N_{t}$ is indicative of the level of crop damage, because every individual that survives to reproductive maturity has completed larval development on plants.

Equation 11 is unrealistic at very high densities (where limited resources would cause density-dependent mortality) or very low densities (where rare insects could fail to find mates and stochastic effects would be observed, and our model can yield fractions of insects rather than whole numbers). However, this measure of relative population size is useful because it illustrates trends in pest densities.

Seasonality. It has been observed that the resistance trait can reduce emergence from diapause (Alyokhin and Ferro 1999, Carrière et al. 2001b, Bird and Akhurst 2004). To represent the effects of winter on a pest in which resistance is fully recessive, we apply further fitness penalties to larvae in every $n$th generation, where the insect goes through $n$ generations in a year. One factor reduces survival of all genotypes, and another factor further reduces survival only of resistant $\mathrm{rr}$ genotypes to represent an overwintering cost associated with the resistant phenotype (e.g., impairing the ability to enter and emerge from diapause or the timing of those). The average relative fitness of larvae of genotype $i$ in winter generations is then

$$
\Omega_{i} \times[\text { overwinter survival] for } i \neq r r
$$

\section{$\Omega_{r r} \times$ [overwinter survival]}

\section{$\times$ [relative overwinter survival of $r r]$ for $r r$}

Simulations. We assume the RIDL construct is fully dominant and completely lethal to females (but not to males), so for $L w$ females $\varepsilon_{\text {female }}=1$ and average fitness $\Omega_{i}=0$, irrespective of $\Phi, \omega_{i}$, and $v_{i}$. Under these conditions, $L L$ homozygotes do not arise in the field and there are seven male and three female viable genotypes defined at the $L / w$ and $s / r$ loci. The LLss male genotype is the engineered released RIDL male, and the other six male genotypes (Lwss, Lwsr, Lwrr, wwss, wwsr, and wwrr) and three female genotypes (wwss, wwsr, and wwrr) arise from matings in the field. In these simulations, $\varepsilon_{\text {male }}$ was set to 0.1 for $L w$ male larvae, to allow for a modest fitness penalty that might be associated with the RIDL insertion (e.g., due to insertional mutagenesis and/or expression of the marker gene; Marrelli et al. 2006).

We explore the effects of release of RIDL males by simulating different combinations of $v$ and $\omega$ representing varying strength and fitness costs of resistance, over a range of refuge sizes $(0-1$ or a subset thereof) and release ratios from 1:1000 (one RIDL male per thousand males in the wild, 0.001 ) to $25: 1$ (or a subset thereof). We simulate the frequencies of all genotypes over 2,500 generations when estimating the value of an equilibrium allele frequency; 200 generations when exploring population dynamics and seasonality; and 500 generations when estimating minimum refuge 

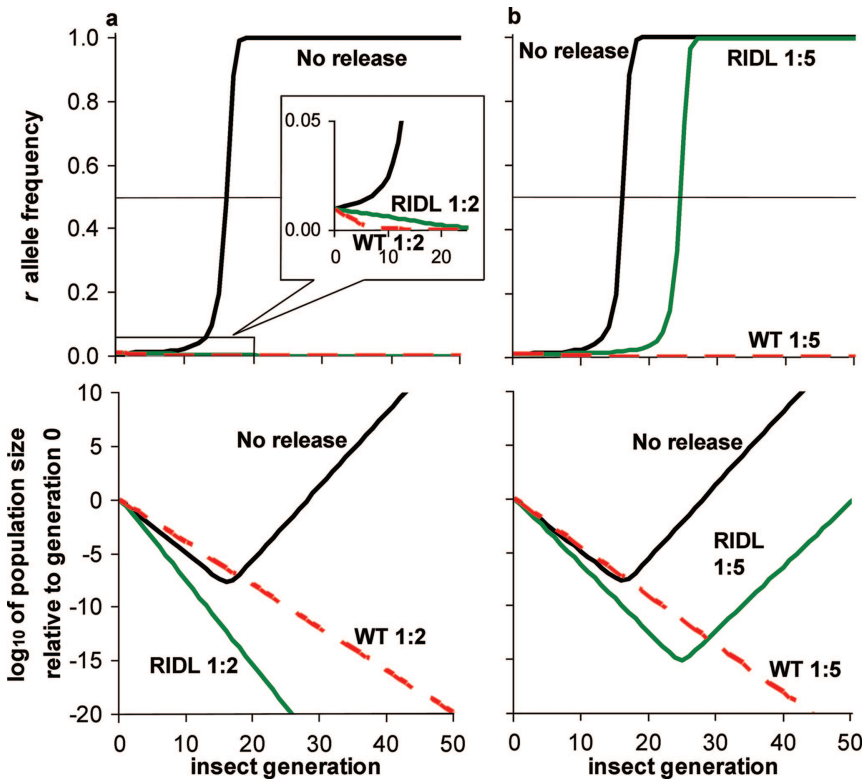

Fig. 1. RIDL release acts in synergy with Bt crops, and wild-type release is of variable relative merit. The top graphs show the frequency of the $r$ allele in naturally emerging adults, and the bottom graphs show the relative population size, over time, for no release of insects (black lines), RIDL release (green lines), and wild-type (WT) release (dashed red lines) with release ratio (a) 1:2, i.e., one RIDL male or WT adult (male or female in equal numbers) per two males in the wild or (b) 1:5. Genotype relative fitness values represent a generic pest for which Bt plants are high-dose and resistance is recessive: in refuge, $v_{s r}=$ $0.9, v_{r r}=0.7$; on Bt plants, $\omega_{s s}=\omega_{s r}=0, \omega_{r r}=0.6$. Initial $r$ allele frequency $p_{0}$ is 0.01 . Offspring per female $2 R_{0}=16$. Refuge is $4 \%$. (Online figure in color.)

sizes for the $r$ allele not to reach 0.5 frequency in the period simulated, for pests for which Bt crops are not high dose. These durations are far beyond the realistic practical lifetime of any single insecticidal crop product, but we use them to illustrate the direction of change of $r$ allele frequency (which can take a long time to become apparent especially where it is initially very rare) and to facilitate estimation of long-term results such as an equilibrium value toward which the $r$ allele frequency will tend.

A standard genetic criterion for the time to evolve resistance is the number of generations taken for $r$ allele frequency to exceed 0.5 (Comins 1977, Tabashnik et al. 2008); this is deemed to indicate a "resistance event." In some circumstances, the allele can tend to an internal equilibrium frequency (between 0 and 1 ), so this criterion can make a seemingly arbitrary distinction between a resistance management regime deemed unsuccessful because the $r$ allele settles just above 0.5 and a regime deemed adequate because the $r$ allele settles just below 0.5 . However, there seems to be a strong connection between the effect on population size and the $r$ allele exceeding 0.5 (see Results, including Fig. 1), so the distinction is relevant. For any given set of parameter values, trial and error can be used to ascertain the minimum refuge size for the $r$ allele not to reach 0.5 frequency. For multiple datasets, the approach was automated to approximate the minimum refuge size needed to prevent the $r$ allele frequency reaching 0.5 during the generations simulated. (Alternative cut-off points such as frequency 0.3 give the same conclusions on relative times to resistance when comparing scenarios; we use 0.5 because of its common acceptance and the clear impact on population size shortly after that value is reached.)

Conditions favoring resistance (e.g., high $r$ allele frequency, nonrecessive resistance and low fitness costs) are more likely to warrant extra measures such as RIDL to delay resistance. For such situations, we parameterized the model using published data for pest insects (Table 1 ), adapting values as appropriate to the aspect under investigation.

We modeled initial allele frequency $p_{0}$ of 0.01 or 0.001 . Although for many pests, $r$ allele frequencies are of the order of $10^{-3}$ based on field data (Gould et al. 1997, Tabashnik et al. 2005, Stodola et al. 2006, Huang et al. 2007, Mahon et al. 2007), the frequency has increased substantially in some field populations of $H$. zea (Ali et al. 2006, Tabashnik et al. 2008) and $H$. armigera (Liu et al. 2008) and resistant fall armyworm have caused Bt corn crop failure (Reynolds 2007), so higher values could be relevant to situations where a RIDL strategy might be implemented. Overestimating the $p_{0}$ value is likely to underestimate potential benefits of RIDL releases implemented at lower allele frequencies; for fully recessive resistance with given fitness parameters, we previously showed that a higher value of $p_{0}$ would need a higher RIDL release ratio to prevent the $r$ allele from spreading (Alphey et al. 2007b).

For fully recessive resistance and $p_{0}=0.001$ (reflecting field data), we showed previously that a re- 
Table 1. Empirical data used to parameterize the model, for pests of Bt CrylAc cotton

\begin{tabular}{|c|c|c|c|}
\hline Parameter & Pest species & Values estimated from empirical data & References \\
\hline \multirow[t]{3}{*}{$\begin{array}{l}\text { Relative fitness on non-Bt plants } \\
\quad(v) \text { and Bt crops }(\omega)\end{array}$} & P. gossypiella & $\begin{array}{l}v_{s s}=v_{s r}=1, v_{r r}=0.485, \omega_{s s}=\omega_{s r}=0 \\
\omega_{r r}=0.46 \times v_{r r}=0.2231\end{array}$ & $\begin{array}{l}\text { Tabashnik et al. (2000), Carrière et al. } \\
\quad(2001 \mathrm{a}, 2005 \mathrm{~b}) \text {, Liu et al. (2001) }\end{array}$ \\
\hline & H. zea & $\begin{array}{l}v_{s s}=v_{s r}=v_{r r}=1 \\
\quad \omega_{s s}=0.1, \omega_{s r}=0.351, \omega_{r r}=0.404\end{array}$ & Tabashnik et al. (2008) \\
\hline & H. armigera & $\begin{array}{l}v_{s r}=0.925, v_{r r}=0.515, \omega_{s s}=0.047 \\
\quad \omega_{\mathrm{sr}}=0.121, \omega_{r r}=0.293\end{array}$ & Tabashnik et al. (2008) \\
\hline Dominance of resistance $(h)$ & H. zea & 0.826 & $\begin{array}{l}\text { Calculated from the fitness values } \\
\text { above (Tabashnik et al. (2008)) }\end{array}$ \\
\hline $\begin{array}{l}\text { Dominance of fitness costs of } \\
\text { resistance }(g)\end{array}$ & H. armigera & 0.155 & $\begin{array}{l}\text { Calculated from the fitness values } \\
\text { above }\end{array}$ \\
\hline Reproductive no. $\left(R_{0}\right)$ & P. gossypiella & 8.6 (we use $2 R_{0}=17$ ) & Liu et al. (2001) \\
\hline Generations per yr & P. gossypiella & 5 & Tabashnik et al. 2008 \\
\hline Overwinter survival & P. gossypiella & 0.05 & Gutierrez and Ponsard (2006) \\
\hline $\begin{array}{l}\text { Relative overwinter survival of } \\
\text { resistant }(\mathrm{rr}) \text { individuals }\end{array}$ & P. gossypiella & 0.29 & Carrière et al. (2001b) \\
\hline
\end{tabular}

We use data on pink bollworm to parameterize our model when comparing the evolution of $r$ allele frequency and population size under alternative assumptions of no seasonality or overwintering. We use $H$. zea data to investigate the effect on the evolution of $r$ allele frequency of varying dominance of resistance. We use $H$. armigera data to assess the effect on $r$ allele frequency of varying the dominance of fitness costs of resistance.

lease ratio as low as 1:4 could justify a refuge as small as $2-3 \%$ of the size required without RIDL release (Alphey et al. 2007b). In this study, we generally simulated release ratios (5:1 and below) lower than those typically employed in an SIT program aimed at pest suppression or elimination. Because we were interested in assessing RIDL release combined with smaller refuges, for most purposes we modeled refuge sizes lower than are typically observed (e.g., the statewide mean refuge size for $\mathrm{Bt}$ cotton in Arizona is $\approx 50 \%$, varying from 14 to $78 \%$ per county; Carrière et al. $2005 \mathrm{~b}$ ). We generally used 4 or $5 \%$ refuge, noting that these were the minimum options for mandatory Bt cotton refuge in the USA pre- and post-2001, respectively. When addressing questions of dominance, we used empirical data for pests with relevant resistance characteristics and considered refuge sizes appropriate to those. For dominance of resistance, we modeled 39\% refuge, as estimated for Arkansas and Mississippi where field-evolved resistance to CrylAc cotton has been detected in H. zea (Tabashnik et al. 2008), $20 \%$ refuge, one of the options under U.S. requirements, and 5\% refuge. Decreased $H$. armigera susceptibility to CrylAc has not been observed in the field, likely due to large effective refuge sizes $(70 \%$ or more in Australia and 87-95\% in China; Tabashnik et al. 2008), except for one report where the effective refuge size is $32 \%$ (Liu et al. 2008). Unsurprisingly, when we modeled $70 \%$ refuge with even a modest RIDL release, we found that the $r$ allele was driven extinct in most cases. To explore fully the effects of varying fitness costs, and for consistency, we again modeled 39,20 , and $5 \%$ refuge.

\section{Results}

The $r$ allele never reaches fixation with RIDL release because of the dilution effect of adding new $s$ alleles at each generation, but the equilibrium frequency can be very close to 1 . Simulations reveal that for any given set of parameter values there is a critical release ratio, above which the $r$ allele tends to extinction. This critical value depends on the genotypes relative fitness values, the initial allele frequency and the refuge size. We conclude that extending the model to RIDL releases alters the critical refuge sizes (equations 3 and 4 ) by the inclusion of terms incorporating the RIDL release ratio and $p_{0}$. If we were able to express these modified formulae explicitly, we could in principle rearrange them to show critical values for any element while keeping the others constant. Instead of determining critical $\Phi$ keeping all else constant, we might thus determine the critical value for RIDL release ratio (for given $p_{0}$, refuge size and fitness values) or derive a critical value for initial frequency $p_{0}$ (for given RIDL release ratio, refuge size and fitness values). We therefore begin by considering the critical release ratio and its relationship with refuge size and initial $r$ allele frequency. Resistance is generally thought to be recessive for most pests, so we start by analyzing that situation. Later, we consider the full range of dominance of resistance or of fitness costs.

Where resistance is recessive $\left(\Phi_{1}=1\right.$, equation 3$)$ but fitness costs are not $\left(\Phi_{2}<1\right.$, equation 4$)$ without releases the $r$ allele will go to extinction or fixation depending on the refuge size and initial allele frequency $\left(\Phi_{2}<\Phi_{1}\right.$, and there is one critical refuge size at $1-\Phi_{3}$, which depends on the value of $\left.p_{0}\right)$. We considered such cases where resistance would not go extinct with no release, and assessed which factors influence the evolution of $r$ allele frequency.

The equilibrium $r$ allele frequency decreases with increasing RIDL release ratio (see example in Table 2 ). The release ratio affects the equilibrium value because it affects the level of dilution by $s$ alleles from released insects that prevents the $r$ allele reaching fixation. This value does not decrease smoothly from close to one (for a very small RIDL release ratio) down to 0 (at or above the critical release ratio) but jumps discontinuously from a positive level to zero. This threshold nonzero equilibrium value could not be determined analytically. With parameters as in Table 
Table 2. Resistant allele equilibrium frequency varies with RIDL release ratio, with a large discontinuity at the threshold. These results were obtained from simulations with the following parameter values: relative fitness on non-Bt plants, $v_{s s}=1, v_{s r}=$ $0.9, v_{r r}=0.7$; relative fitness on Bt plants, $\omega_{s s}=\omega_{s r}=0, \omega_{r r}=$ 0.6 ; refuge size, $1-\Phi=0.05$ (5\% refuge); and initial $r$ allele frequency, $p_{0}=0.01$

\begin{tabular}{|c|c|c|c|}
\hline $\begin{array}{c}\text { RIDL release } \\
\text { ratio } \\
\text { (released } \\
\text { RIDL males } \\
\text { per male in } \\
\text { the wild) }{ }^{a}\end{array}$ & $\begin{array}{c}\text { Equilibrium } r \\
\text { allele } \\
\text { frequency } \\
\text { (to } 5 \text { d.p.) }\end{array}$ & $\begin{array}{c}\text { Time to } \\
\text { exceed } 0.5 \\
\text { frequency } \\
\text { (generations) }\end{array}$ & $\begin{array}{c}\text { Time to } \\
\text { below } 10^{-5} \\
\text { frequency } \\
\text { (generations) }\end{array}$ \\
\hline 0 (no release) & 1 & 27 & $\mathrm{~N} / \mathrm{A}$ \\
\hline 0.01 & 0.99982 & 28 & $\mathrm{~N} / \mathrm{A}$ \\
\hline 0.05 & 0.99911 & 35 & $\mathrm{~N} / \mathrm{A}$ \\
\hline 0.08269972 & 0.99853 & 188 & $\mathrm{~N} / \mathrm{A}$ \\
\hline 0.08269973 & 0 & $\mathrm{~N} / \mathrm{A}$ & 239 \\
\hline 0.1 & 0 & $\mathrm{~N} / \mathrm{A}$ & 94 \\
\hline 0.2 & 0 & $\mathrm{~N} / \mathrm{A}$ & 70 \\
\hline 0.5 & 0 & $\mathrm{~N} / \mathrm{A}$ & 51 \\
\hline
\end{tabular}

${ }^{a}$ Expressed as a decimal, e.g., 0.5 released males per male in the wild is equivalent to ratio $1: 2$ and 0.01 is equivalent to $1: 100$.

2 , the equilibrium allele frequency drops from $\approx 0.999$ to zero on passing a critical RIDL release ratio just below $0.083: 1$. An allele that is heading to extinction will decline more quickly with a higher RIDL release ratio. If the outcome is a nonzero equilibrium, increasing the RIDL release ratio slows the approach to that. With parameters as in Table 2, the $r$ allele frequency would reach 0.5 in 27 generations (from $p_{0}=0.01$ ) with no release but would become $<0.5 \times 10^{-3}$ in that period (and negligible $<10^{-5}$ within 51 generations) with RIDL release of $1: 2$.

The value of the equilibrium does not vary with $p_{0}$ (see example in Table 3). There is a threshold switch effect; all else being equal, for a given level of RIDL release the value of $p_{0}$ determines whether the $r$ allele goes to extinction or toward a fixed equilibrium value, which can be close to 1 . Lowering $p_{0}$ without passing that threshold slows the approach to the nonzero equilibrium if that is the outcome. If the allele is going

Table 3. Initial $r$ allele frequency acts as a switch between two possible outcomes but does not determine the value of a nonzero equilibrium frequency. These results were obtained from simulations with the following parameter values: relative fitness on non-Bt plants, $v_{s s}=1, v_{s r}=0.9, v_{r r}=0.7$; relative fitness on Bt plants, $\omega_{s s}=\omega_{s r}=0, \omega_{r r}=0.6$; refuge size, $1-\Phi=0.05$ (5\% refuge); and RIDL release ratio, 0.2 (1:5 released RIDL males to males in the wild)

\begin{tabular}{lccc}
\hline $\begin{array}{c}\text { Initial } r \\
\text { allele } \\
\begin{array}{c}\text { frequency } \\
p_{0}\end{array}\end{array}$ & $\begin{array}{c}\text { Equilibrium } r \\
\text { allele } \\
\text { frequency } \\
\text { (to 5 d.p.) }\end{array}$ & $\begin{array}{c}\text { Time to exceed } \\
\text { 0.5 frequency } \\
\text { (generations) }\end{array}$ & $\begin{array}{c}\text { Time to below } \\
10^{-5} \\
\text { frequency } \\
\text { (generations) }\end{array}$ \\
\hline 0.2 & 0.99645 & 2 & N/A \\
0.1 & 0.99645 & 3 & N/A \\
0.05 & 0.99645 & 5 & N/A \\
0.01153776 & 0.99645 & 137 & N/A \\
0.01153775 & 0 & N/A & 178 \\
0.01 & 0 & N/A & 70 \\
0.005 & 0 & N/A & 53 \\
0.001 & 0 & N/A & 36 \\
\hline
\end{tabular}

N/A, not applicable.
Table 4. Resistant allele equilibrium frequency varies with refuge size, with a large discontinuity at the threshold. These results were obtained from simulations with the following parameter values: relative fitness on non-Bt plants, $v_{s s}=1, v_{s r}=0.9, v_{r r}=0.7$; relative fitness on Bt plants, $\omega_{s s}=\omega_{s r}=0, \omega_{r r}=0.6$; initial $r$ allele frequency, $p_{0}=0.01$; and RIDL release ratio 0.2 (1:5 released RIDL males to males in the wild)

\begin{tabular}{lccc}
\hline \hline $\begin{array}{c}\text { Refuge size } \\
(1-\Phi)\end{array}$ & $\begin{array}{c}\text { Equilibrium } r \\
\text { allele } \\
\text { frequency } \\
\text { (to 5 d.p.) }\end{array}$ & $\begin{array}{c}\text { Time to } \\
\text { exceed 0.5 } \\
\text { frequency } \\
\text { (generations) }\end{array}$ & $\begin{array}{c}\text { Time to } \\
\text { below } 10^{-5} \\
\text { frequency } \\
\text { (generations) }\end{array}$ \\
\hline $0 \%$ (all plants are Bt) & 1 & 1 & N/A \\
$2 \%$ & 0.99862 & 9 & N/A \\
$4 \%$ & 0.99719 & 25 & N/A \\
$4.368768 \%$ & 0.99692 & 137 & N/A \\
$4.368769 \%$ & 0 & N/A & 212 \\
$5 \%$ & 0 & N/A & 70 \\
$10 \%$ & 0 & N/A & 57 \\
$20 \%$ & 0 & N/A & 55 \\
\hline
\end{tabular}

extinct, lowering $p_{0}$ hastens its demise. Starting a release program sooner (at lower $r$ allele frequency) therefore gives a better resistance management outcome. It seems intuitively reasonable that modest changes in this initial condition do not change the value of the outcome but do affect the time it takes to get close to it.

The effect of the refuge size is qualitatively similar to that of the RIDL release ratio. There is a critical refuge size above which the $r$ allele will tend toward extinction, with the allele approaching an internal equilibrium if the refuge is too small, and a potentially large discontinuity at that threshold. A larger refuge increases the rate of approach to extinction or slows the approach to an internal equilibrium (see example in Table 4).

The frequency and fitness properties of resistant alleles are impossible to predict in advance and difficult to measure accurately in the field even when present. The significant threshold effects mean that a comfortable margin of error would be advisable in setting release ratios and refuge sizes.

Population Control and Resistance Management. The frequency of a spreading $r$ allele follows a classic sigmoid pattern: a relatively long period with little change (the length depending on the starting point $p_{0}$ ), followed by a period of rapid spread, then leveling off approaching the equilibrium frequency, which is usually fixation (Fig. 1). The decline of an $r$ allele due to selection pressure tends to be slower, again following classic resistance evolution patterns, with monotonic decrease (and little apparent change if starting from a low frequency). These general patterns are also seen in the presence of insect releases (Fig. 1). Although the $r$ allele cannot go to fixation, it might get very close to it.

RIDL release introgresses $s$ alleles into the population and contributes to reducing the population size through the female-lethal effect, so it is always more effective at both managing resistance and reducing the pest population than the no release strategy (Bt crops and refuge alone) for any given refuge size. The relative performance of WT and RIDL strategies de- 
pends on whether both (Fig. 1a), WT only (Fig. 1b) or neither (not shown) control resistance.

If the release ratio is sufficient (for the refuge and fitness values) that both RIDL and WT release strategies reverse the spread of resistance (Fig. 1a, top), RIDL release is better at suppressing the population than WT release and no release (Fig. 1a, bottom). (For parameters as in Fig. 1, the minimum sufficient release ratio is 0.2916 , i.e., a little $>1: 3.5)$. The direction of change in population size depends on the population growth rate. With relatively low growth both release regimes can reduce the population size (Fig. 1a). For some higher values of population growth rate, population growth is only slowed not reversed with WT release, but the population decreases with RIDL release (e.g., with parameter values as in Fig. 1a, this happens where $2 R_{0}>40$ instead of 16 , but with $p_{0}=$ 0.05 and $20 \%$ refuge it occurs with $2 R_{0}=16$ ). With very high growth rates, the pest densities can increase under all three regimes.

WT releases are more effective than RIDL releases at managing resistance because $s$ alleles are inherited by both male and female progeny of released insects, not just through the male line as with RIDL insects. Release of wild-type insects, including fertile females, contributes to pest population growth. However, this effect can be mitigated by the resistance management aspect; by reversing the spread of resistance, releases help the Bt crops to be more effective at population suppression. If WT releases control resistance, they initially cause the population to decline more slowly than with no release, but after resistance becomes substantial ( $>0.5$ frequency), with no release the population quickly starts to grow and no release becomes the worst strategy in the long-term (Fig. 1a and b).

If the release ratio is too low, neither strategy controls resistance (for parameters as in Fig. 1, this occurs for release ratios $<0.0750$, i.e., 1:13.3). Population sizes are higher with inadequate WT release than with insufficient RIDL release in the shorter term (while the $\mathrm{Bt}$ crops are still reducing the pest population). After resistance becomes substantial under RIDL release, populations may become larger than they would with WT release. If there is only a short period before resistance becomes widespread under WT release too, this effect might only be temporary and in the longer term (after resistance is widespread) population sizes are again lower with RIDL releases than with WT releases. (For example, we observed such effects with higher $p_{0}$ and larger refuge than in Fig. 1.) However, if resistance spreads significantly later with WT release than with RIDL release, the further population decline during that period may be too great for the female-lethal effect of the RIDL strategy to catch up with over a reasonable timeframe. (We observed this with parameters as in Fig. 1 and smaller release ratios.) Again, the population growth rate can affect whether the population ultimately grows or declines. In situations where neither strategy controls resistance, it is possible after resistance has spread for the population to decline with RIDL releases but increase with WT releases (e.g., with parameter values as in Fig. 1b except with $p_{0}=0.05,20 \%$ refuge and low growth rate $\left.2 R_{0}=4\right)$.

For a small range of release ratios, WT release reverses the spread of resistance whereas RIDL release only slows its spread and prevents it reaching fixation (Fig. 1b). By controlling resistance, the combination of WT release and Bt crops reduces the pest population effectively. With the other strategies, the population starts to grow again after resistance becomes substantial. The value and spread of release ratios for which this situation arises depends on the $r$ allele frequency, fitness parameters and refuge size. With parameters as in Fig. 1, it occurs with release ratios from $0.075(\approx 1: 13.3)$ to $0.2916(\approx 1: 3.5)$ (with $p_{0}=$ 0.001 and $0.5 \%$ refuge, the range is roughly $1: 29-1: 8$ with $p_{0}=0.1$ and $15 \%$ refuge, it is $\approx 1: 3$ to nearly $2.5: 1$ ).

We have not modeled in detail the situation where RIDL genetic construct is not fully lethal $\left(\varepsilon_{\mathrm{female}}<1\right)$. In that case, the performance of the RIDL insects would be intermediate between the fully lethal RIDL $\left(\varepsilon_{\text {female }}=1\right)$ and WT $\left(\varepsilon_{\text {female }}=0\right)$ releases as modeled above, with a similar trade-off between population suppression and resistance management.

We stress that the proportional release policy inherent in our model is not the only possibility. For example, an alternative would be to release a constant number of RIDL males or wild type insects at every generation, rather than a number proportional to the number of males in the current population. If the number released in the first generation were the same, the constant policy would have a greater impact than the proportional policy, but success would be more expensive, as the release numbers do not decline with the population size. However, the constant policy would have an advantage in terms of monitoring; a limitation of the proportional policy is that the adult male population at each generation should be known or predictable (but apart from pink bollworm, Pectinophora gossypiella (Saunders), in recent years, populations of Bt crop pests are generally highly variable in space and time), which would complicate implementation in practice.

RIDL release could be used for pest control in its own right, being a modified version of the SIT, and higher release ratios improve its effectiveness for resistance management. Together, these features show that RIDL is a potential tool for remedial action where a resistance event has occurred. Simulations confirmed that RIDL release can reverse the spread of resistance even from very high frequencies (for parameter values in Fig. 2a, RIDL release at 10:1 can reverse the spread of resistance, although with a refuge of only $15 \%$ it takes $>30$ generations to return to a low value). Where $p_{0}$ exceeds 0.5 , RIDL release might cause the $r$ allele to decline to an internal equilibrium value rather than to extinction. If the combination of release ratio and refuge size are not sufficiently high, this equilibrium value can be above 0.5 and so the resistance problem would not have been dealt with (Fig. 2b), or the rate of decline to extinction can be too slow to be of practical use. For the extensive resistance modeled in Fig. $2 \mathrm{~b}$, increasing the release 

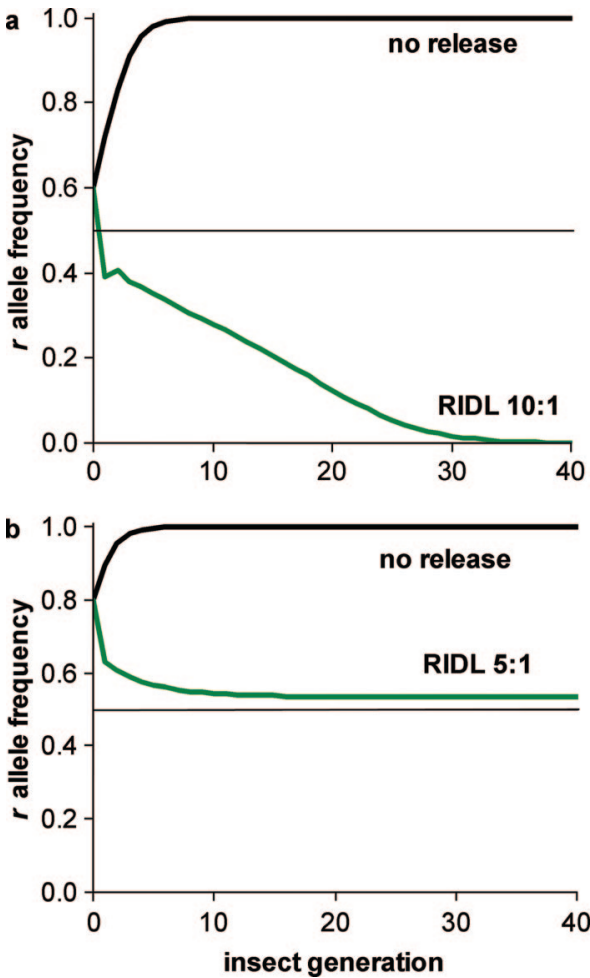

Fig. 2. RIDL release can "clean up" extensive resistance, provided release ratio and refuge are sufficiently high. The graphs show the frequency over time of the $r$ allele for no release (black lines) and for RIDL release (green). (a) Relative fitnesses are as follows: in refuge, $v_{s r}=0.9, v_{r r}=0.4$; on Bt plants, $\omega_{s s}=0, \omega_{s r}=0, \omega_{r r}=0.3$. Initial $r$ allele frequency $p_{0}=0.6$. Refuge $15 \%$. (b) Relative fitness values, representing nonseasonal pink bollworm data: in refuge, $v_{s r}=0.9, v_{r r}=$ 0.485; on Bt plants, $\omega_{s s}=0, \omega_{s r}=0, \omega_{r r}=0.223 . p_{0}=0.8$. Refuge $10 \%$. (Online figure in color.)

ratio from 5:1 to 10:1 would result in $r$ allele frequency 0.37 ; also increasing the refuge from 10 to $20 \%$ refuge would reduce the frequency below 0.01 in 27 generations.

Seasonality. Although Bt crops control pest densities initially, the population would increase rapidly after resistance is established, especially in an environment with no seasonal effects, whereas RIDL release could suppress the population effectively and local elimination might result (Figs. 1 and 3). Where larvae in each relevant generation incur costs of overwintering, the population suffers a step reduction once in every year (Fig. 3). These steps can be seen in the population curves for both no release and RIDL release, but an overall trend in pest numbers is clear and dominates the results more than the in-year variation.

Winter mortality helps to suppress the population. For example, with seasonal parameter values for pink bollworm (Table 1), although the efficacy of Bt crops is significantly reduced after resistance arises, it seems to remain just good enough to keep pest densities in check (Fig. 3). If individuals harboring one or more $r$
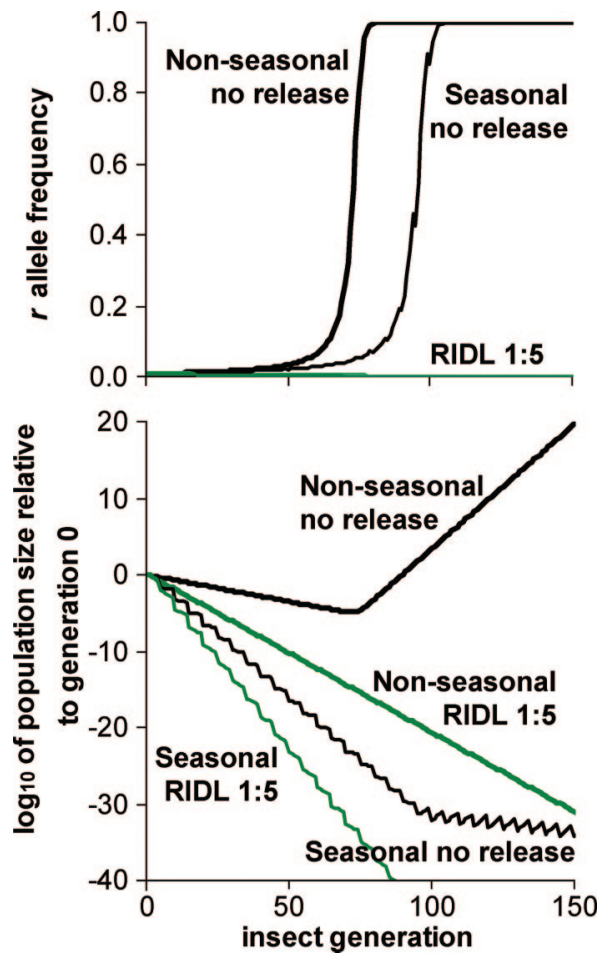

Fig. 3. Winter slows resistance evolution and population growth but does not significantly alter the relative benefits of RIDL release. The top graph shows the frequency of the $r$ allele, and the bottom graph shows the relative population size, over time, for no release of insects (black lines) and for a RIDL release ratio of 1:5 (green). Parameter values represent pink bollworm: relative fitness in refuge, $v_{s r}=1, v_{r r}=$ 0.485; on Bt plants, $\omega_{s s}=\omega_{s r}=0, \omega_{r r}=0.223$; winter occurs every fifth generation, overwinter survival $0.05, \mathrm{rr}$ relative overwinter survival 0.29 ; offspring per female $2 R_{0}=17$. Refuge is $10 \%$. Initial $r$ allele frequency $p_{0}=0.01$. Where conditions are maintained year-round (thick lines), population reduction is slower and resistance management is weaker than where winter imposes survival penalties for all genotypes and extra fitness costs for resistant homozygotes (thinner lines). (Online figure in color.)

alleles die disproportionately in winter, then the allele progresses more slowly toward fixation, or more rapidly toward extinction, than in a nonseasonal scenario, especially bearing in mind that without any winter the insects could have more generations per year (Fig. 3; with no release and $10 \%$ refuge, from $p_{0}=0.1$ the $r$ allele exceeds 0.5 in 73 generations with no seasons but 96 generations with winter). Comparing the two scenarios, the program of RIDL release has a greater effect on the evolution of allele frequency than winter has.

Winter seems to have little effect on the relative benefit of the RIDL strategy. For example, the nonseasonal model with no release, using pink bollworm data, has a critical proportion of Bt crops (equation 4) at 0.698 , i.e., a critical refuge size of $\approx 30 \%$; with a smaller refuge the $r$ allele frequency will increase toward fixation. Simulated RIDL release at 1:5 reduces 
that minimum refuge to $6.4 \%$, only about a fifth (21\%) of the refuge required with no release. When overwintering costs are taken into account, the minimum refuge sizes are $\approx 25 \%$ for no release and $5.5 \%$ for RIDL release, which is also about a fifth $(22 \%)$ of the refuge needed with no release.

Dominance of resistance. With no released insects, we can deduce what effects varying dominance of resistance can have on the equilibrium $r$ allele frequency. Increasing dominance of resistance moves the relative fitness of heterozygotes on Bt plants $\omega_{s r}$ further from $\omega_{s s}$ and closer to $\omega_{r r}$. Altering $\omega_{s r}$ in that manner, while keeping all other fitness values constant, decreases the $\Phi_{1}$ critical threshold for Bt crops (equation 3) (except where costs of resistance are recessive, in which case $\left.\Phi_{1}=0\right)$ and increases $\Phi_{2}$ (equation 4 ) (except where costs are dominant, which forces $\Phi_{2}=0$ ). If the relative fitness values are such that $\Phi_{1}<\Phi_{2}$, greater dominance widens the range of $\Phi$ for which $\Phi_{1}<\Phi<\Phi_{2}$ (where heterozygotes have the overall advantage, i.e., "marginal overdominance"), and the $r$ allele will persist and settle at an internal equilibrium value, in some cases instead of going to extinction (where $\Phi<\Phi_{1}$ with the lower dominance) or to fixation (where previously $\Phi_{2}<\Phi$ ). If $\Phi_{1}>\Phi_{2}$, increasing dominance could change $\Phi_{1}$ and $\Phi_{2}$ sufficiently to reverse that relationship. This would add to the range of potential outcomes by making an internal equilibrium allele frequency possible (which only occurs when $\Phi_{1}<\Phi<\Phi_{2}$ ). In summary, increasing dominance of resistance can change outcomes by altering thresholds and creates more possibilities for the $r$ allele to persist.

We investigated the relationship between the minimum refuge size required to prevent the spread of resistance and the RIDL release ratio. We illustrate this using parameter values estimated for $\mathrm{H}$. zea (Table $1)$ and varying values of $h$. Here, the Bt toxins are not high dose (some ss and $s r$ insects survive). There are no fitness costs of resistance (all genotypes survive equally well in the refuge), so the $r$ allele has no disadvantage and with no releases the favorable selection pressure on $\mathrm{Bt}$ crops will drive it to fixation, whatever the dominance of resistance and whatever the refuge size. First, we consider starting a release program from $p_{0}=0.01$. For all values examined, RIDL male release can reduce the minimum refuge size required (Fig. 4a). Even for very low RIDL release (1:1000), some Bt crops can be grown (refuge $<100 \%$ ), and with higher release ratios the reduction in minimum refuge size is considerable. Compared with fully recessive $r$ alleles, where low RIDL release ratios can have a significant effect on minimum refuge size, for partially dominant or dominant resistance $(h>0)$ the refuge reduction with low release ratios is less substantial.

For any given release ratio up to roughly $1: 10$, a higher refuge is needed to control a more dominant $r$ allele. However, for release ratios rising from $\approx 1: 1$ that effect is reversed (Fig. 4a). For example, if 6.5 RIDL males are released for each male in the wild, the minimum refuge required is $6.1 \%$ for $h=0.1$, and this
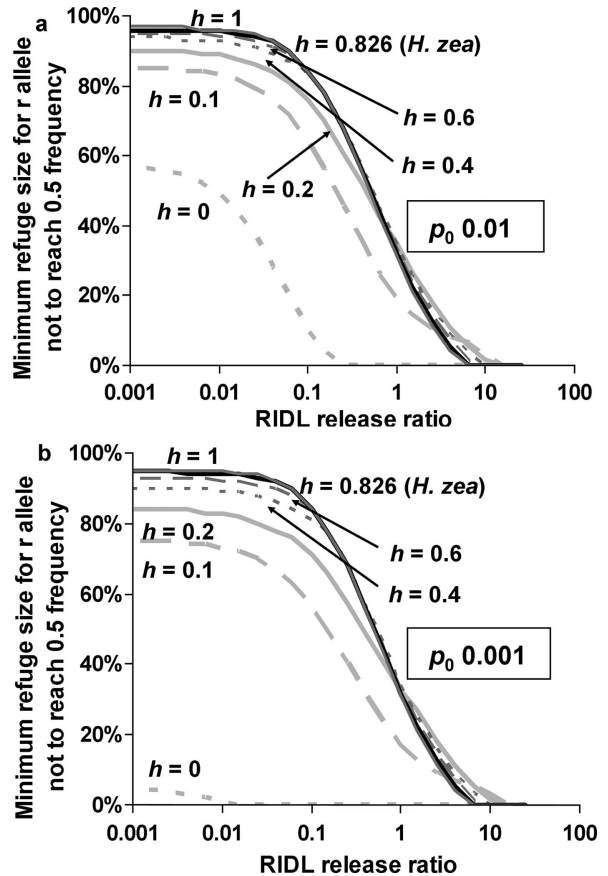

Fig. 4. At low RIDL release ratios, increasing dominance of resistance requires a larger refuge, but at higher RIDL release ratios, a partially reversed effect can be observed. Relative fitnesses, representing $H$. zea data, are as follows: in refuge, $v_{s s}=v_{s r}=v_{r r}=1$; on Bt plants, $\omega_{s s}=0.1, \omega_{s r}=0.1$ to 0.404 (by varying $h$ ), $\omega_{r r}=0.404$. Initial $r$ allele frequency $p_{0}$ is (a) 0.01 or (b) 0.001 . Critical refuge sizes for controlling the $r$ allele, were approximated for a range of RIDL release ratios from 1:1000 to $25: 1$ by determining the minimum refuge size for which the allele frequency does not reach 0.5 in 500 generations. This was repeated for resistance of varying dominance: $h=0$ recessive (light gray dotted line), 0.1 (light gray dashed line), 0.2 (light gray solid line), 0.4 (dark gray dotted line), 0.6 (dark gray dashed line), 0.826 (as reported for $H$. zea, black line), or 1 dominant (dark gray solid line).

decreases through intermediate levels of dominance, down to $0 \%$ (no refuge needed at all) for $h=0.826$ (the actual estimate from $H$. zea data) and $h=1$. This phenomenon could be explained by the genetics of this resistance. There are no fitness costs of resistance, so neither allele has any advantage in the refuge. At low refuge sizes, almost the whole area is planted with Bt crops so very few susceptible $s s$ insects will survive and the evolution of the $s$ allele frequency depends mainly on the heterozygotes, more of which will survive to reproductive maturity if the resistance is more dominant.

The same patterns are observed if releases are started when $p_{0}$ is 0.001 rather than 0.01 (Fig. 4b). Starting the program earlier (Fig. 4, compare a and b) reduces the minimum refuge size considerably for recessive resistance ( $h=0$, light gray dotted lines), and there is a lesser but still noticeable effect for nearly recessive resistance ( $h=0.1$, light gray dashed lines). For more dominant resistance (higher $h$ ), the improvement is slight. 
We investigated the effect of dominance of resistance on the ultimate fate of the $r$ allele for a range of RIDL release ratios, for several refuge sizes (Fig. 5). First, we assumed $39 \%$ refuge (Fig. 5a). With the fitness values adapted from $H$. zea data, the recessive version of the $r$ allele becomes extinct and so do versions with low dominance $h$. There are threshold $h$ values, which rise with increasing release ratio, where the $r$ allele equilibrium jumps from extinction to persistence. The threshold effect is not discontinuous for the relatively higher ratio $(5: 1)$. The $r$ allele persists above 0.5 frequency for lower RIDL release ratios (1:10 and 1:5) and below 0.5 for moderate release ratios (1:1 and 5:1). The equilibrium allele frequency decreases with increasing release ratio, which is consistent with our results for recessive resistance with nonrecessive costs (Table 2). Once over the threshold dominance level, increasing $h$ decreases the equilibrium frequency if it is $>0.5$ and increases it if it is $<0.5$.

At the value estimated for $H$. zea $(h=0.826)$, the $r$ allele goes to fixation with no releases (reaching 0.5 in 19 generations starting from 0.01 frequency), to 0.837 frequency with 1:10 RIDL release ratio (reaching 0.5 in 20 generations), and to 0.745 with 1:5 releases $(0.5$ in 23 generations). RIDL release at 5:1 only achieves a potentially acceptable equilibrium frequency 0.215 . With those fitness values, $39 \%$ refuge and initial allele frequency 0.01 , low levels of RIDL release are an improvement on no release, but the $r$ allele can still become very common fairly rapidly.

Simulations with $p_{0}$ at 0.001 instead of 0.01 produce virtually identical results (indistinguishable by eye from Fig. 5a). The threshold values of $h$ are very slightly higher with lower $p_{0}$, but outside of that small range of $h$ values the eventual allele frequencies are identical. This is consistent with our results for recessive resistance with nonrecessive costs (Table 3); the initial allele frequency acts as a switch between extinction or persistence but does not alter the value of that equilibrium frequency of the persistent allele.

We obtain broadly similar results with a $20 \%$ refuge (Fig. 5b) instead of 39\%. The thresholds are at lower values of $h$ and the persistent allele frequencies are higher. Also, there is a discontinuity in the threshold effect even at 5:1 RIDL release. With 5\% refuge (Fig. $5 \mathrm{c}$ ), the $h$ threshold values are lower still, and above that threshold with 5:1 release ratio the persistent allele frequency is above 0.5 (this consistent with Fig. $4 \mathrm{a}$, which shows that 5:1 ratio is sufficient to prevent the $r$ allele spreading if $h=0$, but not if $h=0.1$ ).

For these simulations with varying dominance of resistance, we have so far assumed no fitness costs of resistance. When we include such costs, we obtain qualitatively similar outcomes (Fig. 5d). The presence of fitness costs creates selection pressure against resistance in the refuge, making it easier to control. With fitness costs (Fig. 5, compare a and d) thresholds are at higher values of $h$ and eventual allele frequencies are lower. As illustrated, with no release the $r$ allele can go extinct at low $h$ (because of the extra selection pressure) and tends to an equilibrium below one at high values of $h$ (where having a second copy of the
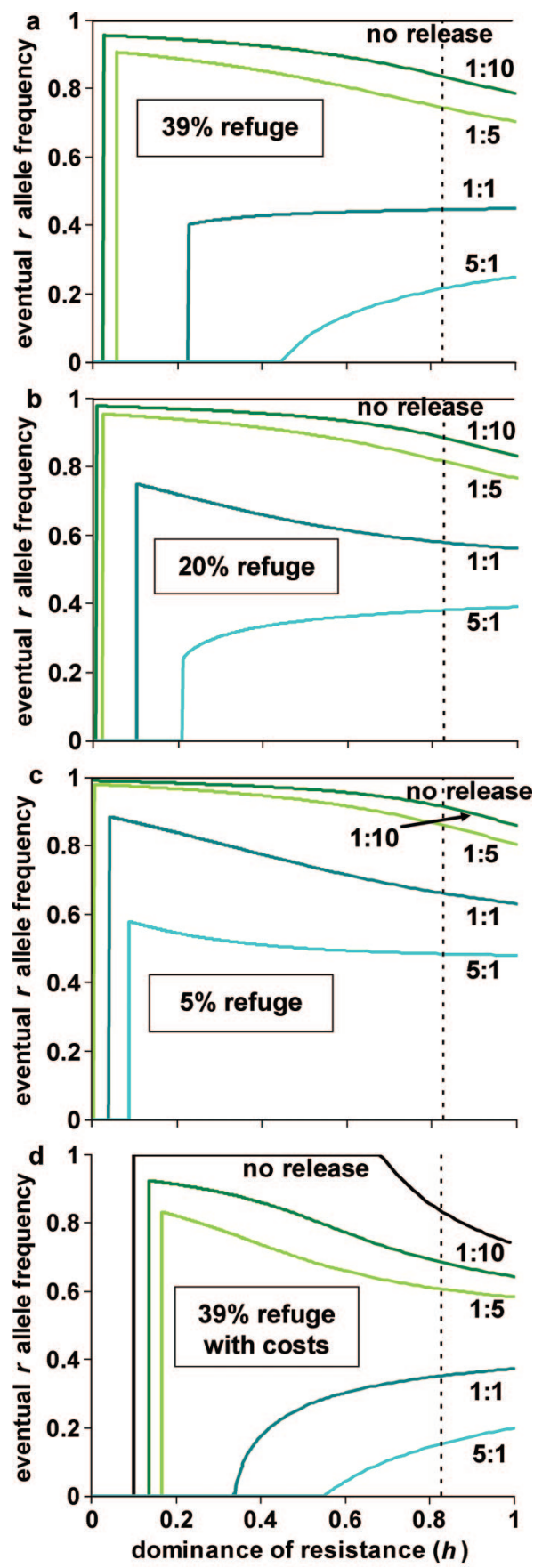

Fig. 5. There are threshold values of dominance of resistance at which the $r$ allele switches from extinction to persistence. The graphs show the equilibrium allele frequency toward which the $r$ allele converges for resistance varying from recessive $(h=0)$ to dominant $(h=1)$. Relative fitness values and $p_{0}$ are as in Fig. 4 a, refuge is (a) $39 \%$, (b) $20 \%$, or (c) $5 \%$, and release ratios are as marked. The dotted line indicates $h=0.826$ as estimated from $H$. zea data. $\mathrm{d}$ is as for a, except there are fitness costs of resistance: in refuge, $v_{s r}=0.95$ and $v_{r r}=0.8$ (instead of 1 ). (Online figure in color.) 


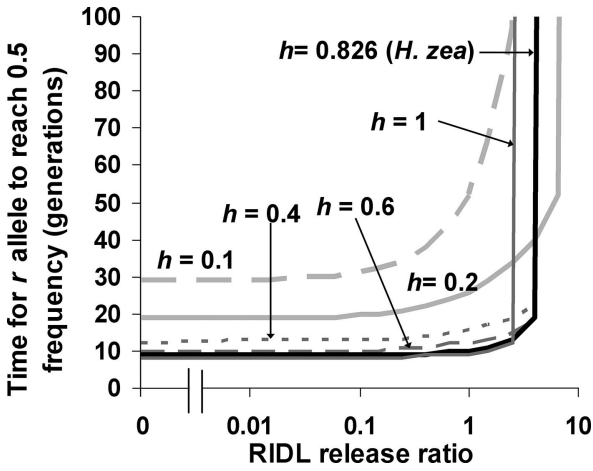

Fig. 6. RIDL releases slow the spread of the $r$ allele, with higher dominance of resistance reducing this slowing effect but also reducing the critical release ratio that is sufficient to stop resistance spreading. The graph shows the number of generations after which the $r$ allele exceeds 0.5 frequency, starting from $p_{0}=0.001$, for resistance of varying dominance as marked: $h=0.1$ (light gray dashed line), 0.2 (light gray solid line), 0.4 (dark gray dotted line), 0.6 (dark gray dashed line), 0.826 (as reported for H. zea, black line), or 1 dominant (dark gray solid line). Relative fitness values are as in Figs. 4 and 5. Refuge is 5\%. Recessive resistance $(h=0)$ is not shown, because the $r$ allele does not reach 0.5 frequency regardless of releases. The RIDL release ratio axis is on a log scale except that the values for 0 (no release) are plotted and joined to those for 0.01 (1:100) by straight lines.

$r$ allele confers little or no advantage but has some extra cost, so the heterozygote has an advantage over the resistant homozygote).

Although the equilibrium value conveys information about the evolution of $r$ allele frequency, the pattern during the early years is of more practical interest. These transient dynamics follow the classic sigmoid curves evident in Figs. 1-3. If the $r$ allele exceeds 0.5 frequency, the population growth rate then increases significantly, as illustrated in Figs. 1 and 3 . We investigated the effect of RIDL release ratio on the time taken to reach 0.5 frequency for a range of values of dominance of resistance. With fitness parameters adapted from $\mathrm{H}$. zea data and $5 \%$ refuge (Fig. 6 ), a recessive $r$ allele $(h=0)$ would not reach 0.5 frequency with no (or any) releases, but with dominance $h=0.1$, the frequency passes 0.5 in 29 generations (from $p_{0}=0.001$ ) with no release and that period is reduced as dominance increases. Very low RIDL releases have little effect on the timing, but at moderate levels they can slow the spread of resistance. This slowing effect is less marked as dominance of resistance increases, although greater dominance $h$ reduces the critical release ratio with which the $r$ allele will not reach 0.5 frequency.

We conclude that for highly dominant resistance the advantages of the RIDL strategy are not as substantial as they are for recessive or nearly recessive resistance. As with other resistance management strategies, more dominant resistance is harder to defeat.
Dominance of Fitness Costs of Resistance. Greater dominance of fitness costs of resistance reduces the relative fitness of heterozygotes in the refuge $\left(v_{s r}\right)$ while keeping all other fitness values constant. We can analyze the situation with no releases. More dominant costs raise the critical proportion of $\mathrm{Bt}$ crops $\Phi_{1}$ (equation 3) (unless resistance is recessive, which forces $\Phi_{1}=1$ ) and lower $\Phi_{2}$ (equation 4) (unless resistance is dominant, which makes $\Phi_{2}=1$ ). These are the opposite of the effects seen for increasing $h$. Generally, increasing dominance of fitness costs creates fewer possibilities for the $r$ allele to tend to an internal equilibrium frequency and more opportunities for extinction.

To illustrate this, consider an example (data for $H$. armigera, Table 1) where the $\mathrm{Bt}$ toxins are not highdose, fitness costs are not very dominant $(g=0.155)$ and the no-release critical proportions of Bt crops are $\Phi_{1}$ and $\Phi_{2}$ (to 3 decimal places) so $\Phi_{1}<\Phi_{2}$. With $70 \%$ refuge $\left(\Phi=0.3\right.$, so $\left.\Phi<\Phi_{1}\right)$, the $r$ allele will decline to extinction. With $39 \%$ refuge $\left(\Phi=0.61\right.$ and $\Phi_{1}<$ $\Phi<\Phi_{2}$ ) the $r$ allele will tend toward an intermediate equilibrium frequency, in this case 0.224 . With a $20 \%$ refuge $\left(\Phi=0.8, \Phi_{2}<\Phi\right)$, the $r$ allele will go to fixation. If we now make the costs of resistance more dominant by lowering heterozygote fitness in the refuge, the critical values would change as described above. With $39 \%$ refuge the $r$ allele equilibrium frequency starts at 0.349 with recessive costs of resistance $(g=0)$, decreases in value with increasing $g$ and reaches 0 when $g$ is 0.239 . (This happens because if $g<0.239$ then $\Phi_{1}<$ $0.61<\Phi_{2}$, if $0.239<g<0.301$ then $0.61<\Phi_{1}<\Phi_{2}$, and if $g>0.301, \Phi_{2}<\Phi_{1}$ and with $p_{0}=0.01 \Phi$ lies below $\Phi_{3}$.) With $20 \%$ refuge and $p_{0}=0.01$, the $r$ allele switches from fixation to extinction when $g$ increases above 0.621 (because $\Phi_{3}$ changes from $<0.8$ to $>0.8$ ) . These outcomes for no releases are confirmed by simulation (black lines in Fig. 8; see below).

According to this analysis, with no releases the lowest refuge size for the $r$ allele represented in this example data $(H$. armigera) to tend to extinction is $\approx 49.7 \%$ (so that $\Phi<\Phi_{1}=0.504$ ). Decreasing the refuge below $49.7 \%$ at first results in the $r$ allele tending toward an internal equilibrium and although that remains below 0.5 frequency, the lower refuge size would still satisfy our criterion that the $r$ allele frequency does not reach 0.5 . Simulations show that the minimum refuge size defined this way is $\approx 33.7 \%$. If the fitness costs were instead dominant (make $g=1$ by setting $v_{s r}$ equal to $v_{r r}$ ), that minimum refuge size is $13.6 \%$.

We investigated the relationship between the minimum refuge size required to prevent the spread of resistance (starting from $p_{0}=0.01$ ) and the RIDL release ratio, for various values of $g$ (Fig. 7). RIDL release over $\approx 1: 10$ reduces noticeably the minimum refuge required (at lower ratios RIDL release gives only a marginal improvement on no release). The reduction in minimum refuge size can be considerable and there is more benefit where the costs of resistance are less dominant. When costs of the $r$ allele are less dominant, more heterozygous insects 


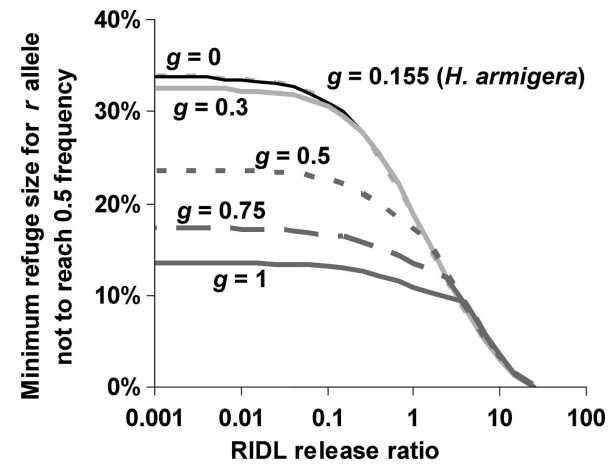

Fig. 7. More dominant fitness costs of resistance reduce the minimum refuge size needed to contain the $r$ allele, although the differences are slight at higher release ratios. Relative fitnesses, representing $H$. armigera data, are as follows: in refuge, $v_{s r}=0.515$ to 1 (by varying $g$ ), $v_{r r}=0.515$; on Bt plants, $\omega_{\mathrm{ss}}=0.047, \omega_{s r}=0.121, \omega_{r r}=0.293$. Initial $r$ allele frequency $p_{0}$ is 0.01 . Minimum refuge sizes for which the $r$ allele frequency does not reach 0.5 in 500 generations were determined for a range of RIDL release ratios from 1:1000 to 25:1. This was repeated for resistance costs of varying dominance: $g=0$ recessive (light gray dotted line, almost entirely obscured beneath black line), 0.155 (as reported for $H$. armigera, black line), 0.3 (light gray solid line), 0.5 (dark gray dotted line), 0.75 (dark gray dashed line), or 1 dominant (dark gray solid line). Note that the vertical axis only extends to 0.4 , not to 1 .

survive in the refuge and the extra $s$ alleles they contribute reduce the effect of introgression of $s$ alleles from released insects. For a given release ratio, a higher minimum refuge is needed to deal with an $r$ allele that has less dominant fitness costs. However, the minimum refuge size varies little for $g$ in the range $0 \leq g \leq 0.3$ across the whole range of release ratios; it is only at higher values of $g$ that the influence of dominance on minimum refuge size becomes obvious. At higher release ratios, the dominance of fitness costs has negligible effect on the minimum refuge size.

RIDL releases lower the equilibrium frequency and lower the critical value of $g$ above which the $r$ allele goes extinct; higher release ratios increase those effects (Fig. 8). With 39\% refuge, the equilibrium allele frequency decreases continuously with increasing $g$ until it reaches zero (Fig. 8a). However, with $20 \%$ refuge, except for RIDL release at $5: 1$, the outcome for the $r$ allele switches discontinuously from persistence to extinction as dominance of resistance costs increases (Fig. 8b). The same kinds of changing outcomes are seen with RIDL releases as we described analytically for no release (above): the nature of the $g$ threshold (continuous or discontinuous effect) can be altered by having different refuge sizes (Fig. 8, compare a and b) or by varying the RIDL release ratio (compare 5:1 to other lines in Fig. 8b). A 5\% refuge is insufficient to prevent the $r$ allele spreading with any release ratio below nearly 10:1 (Fig. 7), so there is no threshold effect and the dominance of fitness costs has only a
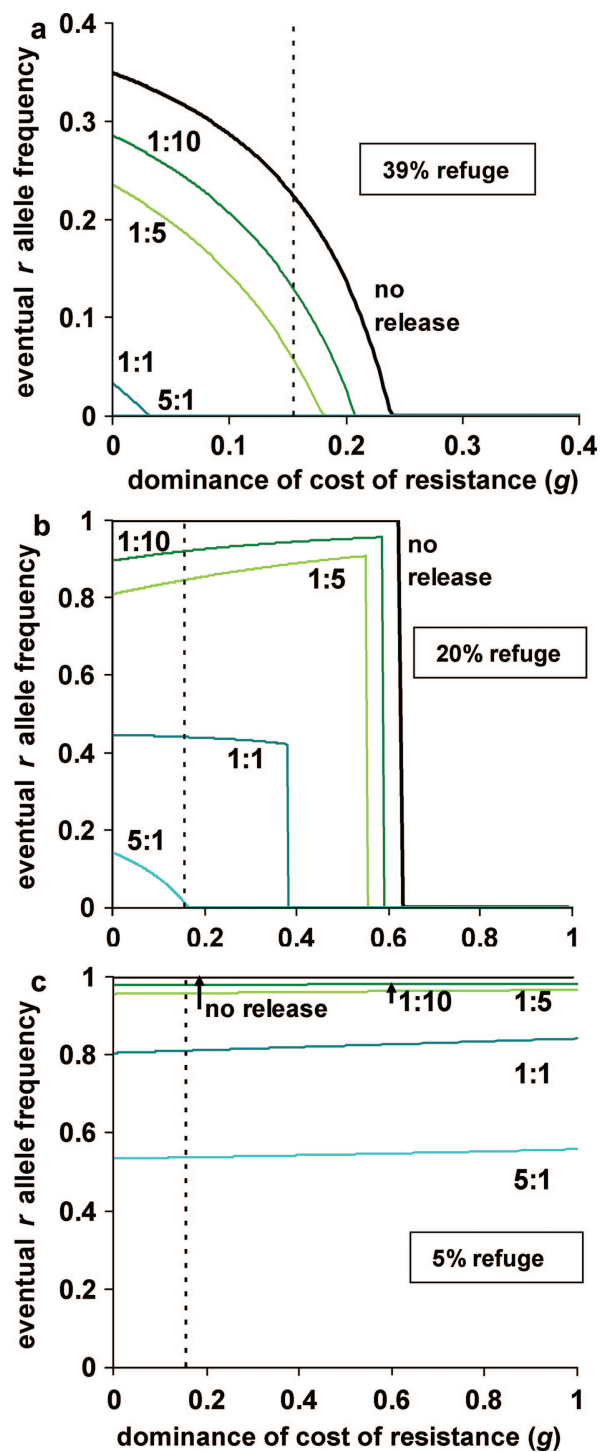

Fig. 8. The equilibrium $r$ allele frequency decreases with increasing dominance of fitness costs of resistance, from persistence to extinction. The graph shows the equilibrium frequency toward which the $r$ allele converges for fitness costs of resistance varying from recessive $(g=0)$ to dominant $(g=1)$. Relative fitness values and $p_{0}$ are as in Fig. 7 and release ratios are as marked. The dotted line indicates $g=$ 0.155 as estimated from $H$. armigera data. Note that panel (a) is on a different scale; axes extend only to 0.4 . With $39 \%$ refuge, (a) there is no discontinuous threshold effect, but with $20 \%$ refuge (b) there is, and with $5 \%$ refuge (c), there are no thresholds. (Online figure in color.)

slight effect on the eventual frequency of the $r$ allele (Fig. 8c).

\section{Discussion}

We have shown that a RIDL approach could combine synergistically with Bt crops to form an effective 
component of a pest management strategy. The effectiveness for managing resistance can be improved by any combination of increasing the RIDL release ratio, commencing releases earlier (when the resistant allele is at a lower frequency) and increasing the refuge size. These measures slow the onset of resistance and may reverse it. Increasing the ratio of released RIDL males to males in the wild also has a beneficial impact on the size of the pest population.

Simulated RIDL release is consistently better than Bt crops and refuge alone for both resistance management and population control. Wild-type release is predicted to be more effective for resistance management than the release of RIDL males. However, release of wild type males and females has a marginally detrimental effect on population size (and therefore on crop damage) compared with no release, until such time as resistance would have become substantial with no releases (likely to be several years for most key pests targeted by Bt crops).

RIDL release could potentially achieve a considerable reduction in the minimum refuge size required to prevent the spread of resistance. The relative benefit seems unaffected by environmental seasonality. The degree of advantage for resistance management depends on the fitness parameters associated with the resistant allele and the allele frequency. Generally, RIDL is of greater benefit in situations that are already more favorable for resistance management - where resistance is rarer, is less dominant or has more dominant fitness costs. Like the high-dose refuge strategy itself, RIDL release is less effective when applied to dominant resistant alleles in pest populations where $\mathrm{Bt}$ plants do not kill all susceptible individuals.

The existence of significant threshold effects arising from multiple parameters highlights the need for good information about the target pest population, when planning a resistance management strategy. The genetic fitness parameters and frequency of rare (possibly nonexistent) resistant alleles are difficult to measure or estimate in practice. It would be advisable to select a release ratio comfortably above the estimated critical value to avoid inaccuracy or fluctuations switching the situation to one where a large frequency could result instead of extinction.

The economic attractiveness of the RIDL strategy depends on an assessment of the costs of a release program compared with the net benefits of refuge costs foregone. For growers, refuges are an inter-temporal economic trade-off; farmers lose the short-term benefits of $\mathrm{Bt}$ technology in the areas planted as refuge in return for extending the period over which Bt plants retain their efficacy (Frisvold and Reeves 2008). Estimates of the costs of refuges vary widely and, although generally agreeing that some refuge is preferable to none, various economic studies have come to inconsistent conclusions about what refuge policies would be optimal for grower returns. Entomological and economic information, both subject to uncertainty, are needed to assess where any particular region's agricultural producers are on the trade-off surfaces charting the longer-term benefits and costs of refuge policies. RIDL release would add an extra dimension to such analyses. The costs of a RIDL release program depend on factors such as pest densities (the number of insects released vary with population size and change over time) and the availability of modest spare capacity in existing SIT facilities. RIDL release is more likely to be implemented as an areawide program and so would be influenced more by regional adoption choices than by individual growers' decisions.

There is evidence that many growers have planted less Bt crops than permitted (Carrière et al. 2005b, Frisvold and Reeves 2008). This suggests that noneconomic factors are also influencing decisions, such as cautious adoption of new technology and a risk-averse attitude if the variance of profits is higher for Bt plants (Frisvold and Reeves 2008). Another possible explanation is a halo effect, where long-term regional pest suppression in areas of high adoption can reduce pest pressure in the region generally, thereby generating benefits for non-Bt plants too and reducing the marginal advantage of planting more Bt crops (Carrière et al. 2003, Frisvold and Reeves 2008). In such circumstances, where mandatory refuge sizes might not actually be binding constraints, a RIDL option with lower permitted refuges may not have an apparent economic benefit.

An economic case for a RIDL approach might be more persuasive in situations where the strategies in current use have failed. Potential short-term responses to a resistance event include additional chemical control, early harvest, SIT releases and biological control (such as parasitic nematode treatments), and remedial action the following year could include temporary cessation of cultivating the relevant Bt crop and upward revision of mandatory refuge sizes (Frisvold and Reeves 2008). RIDL release could be added to this toolkit and, unlike the other options available, it would contribute to both reduction of pest densities and decrease of resistant allele frequencies. For a successful outcome using only RIDL release and refuge manipulation, it would be important to use a sufficiently high combination of refuge and release ratio to bring the resistant allele rapidly down to a low level.

Dynamic selection pressures and rapidly updated and expanding technology make it impossible to formulate a universally optimal resistance management strategy. Instead, it is sensible to adopt a framework within which an appropriate mix of measures can be selected, tailored to the particular genetically modified crop and incorporated toxin(s), and the local pest species, economic factors, environmental conditions and agricultural practices. For example, in the USA, for each approved crop type a few alternative refuge requirements are specified, with some choice as to the position of refuges and whether they may be sprayed, and special cases for particular geographic regions. Within this context, the addition of appropriate RIDL strategies to the menu of options could be a sensible extension to existing crop pest management regimes. 


\section{Acknowledgments}

We thank Paul Coleman for contribution to the underlying theory and simulation model. This work was supported by the UK Biotechnology and Biological Sciences Research Council. M.B.B. is a Royal Society University Research Fellow.

\section{References Cited}

Akhurst, R. J., W. James, L. J. Bird, and C. Beard. 2003. Resistance to the CrylAc $\delta$-Endotoxin of Bacillus thuringiensis in the cotton bollworm, Helicoverpa armigera (Lepidoptera: Noctuidae). J. Econ. Entomol. 96: 12901299.

Ali, M. I., R. G. Luttrell, and S. Y. Young. 2006. Susceptibilities of Helicoverpa zea and Heliothis virescens (Lepidoptera: Noctuidae) populations to CrylAc insecticidal Protein. J. Econ. Entomol. 99: 164-175.

Alphey, L. S. 2007. Engineering insects for the sterile insect technique, pp. 51-60. In M. Vreysen, A. Robinson, and J. Hendrichs [eds.], Area-wide control of insect pests: from research to field implementation. Springer, Dordrecht, The Netherlands.

Alphey, L., D. Nimmo, S. O'Connell, and N. Alphey. 2007a. Insect population suppression using engineered insects. In S. Aksoy [ed.], Transgenesis and the management of vector-borne disease. Landes Bioscience, Austin, TX. (http://www.eurekah.com/chapter/3233).

Alphey, N., P. G. Coleman, C. A. Donnelly, and L. Alphey. 2007b. Managing insecticide resistance by mass release of engineered insects. J. Econ. Entomol. 100: 1642-1649.

Alphey, N., P. G. Coleman, M. B. Bonsall, and L. Alphey. 2008. Proportions of different habitat types are critical to the fate of a resistance allele. Theor. Ecol. 1: 103-115. (doi: $10.1007 /$ s12080-008-0010-8).

Alstad, D. N., and D. A. Andow. 1995. Managing the evolution of insect resistance to transgenic plants. Science (Wash., D.C.) 268: 1894-1896.

Alyokhin, A. V., and D. N. Ferro. 1999. Relative fitness of Colorado potato beetle (Coleoptera: Chrysomelidae) resistant and susceptible to the Bacillus thuringiensis Cry3A toxin. J. Econ. Entomol. 92: 510-515.

Bates, S. L., J. Z. Zhao, A. M. Shelton and R. T. Roush. 2005. Insect resistance management in GM crops: past, present and future. Nat. Biotechnol. 23: 57-62. (doi:10.1038/ nbt1056).

Bird, L. J., and R. J. Akhurst. 2004. Relative fitness of Cry1Aresistant and -susceptible Helicoverpa armigera (Lepidoptera: Noctuidae) on conventional and transgenic cotton. J. Econ. Entomol. 97: 1699-1709.

Brookes, G., and P. Barfoot. 2006. GM crops: the first ten years-global socio-economic and environmental impacts. ISAAA Brief no. 36. International Service for the Acquisition of Agri-biotech Applications, Ithaca, NY.

Caprio, M. A. 2001. Source-sink dynamics between transgenic and non-transgenic habitats and their role in the evolution of resistance. J. Econ. Entomol. 94: 698-705.

Carrière, Y., and B. Tabashnik. 2001. Reversing insect adaptation to transgenic insecticidal plants. Proc. R. Soc. (Lond) B 268: 1475-1480.

Carrière, Y., C. Ellers-Kirk, Y. B. Liu, M. A. Sims, A. L. Patin, T. J. Dennehy, and B. E. Tabashnik. 2001a. Fitness costs and maternal effects associated with resistance to transgenic cotton in the pink bollworm (Lepidoptera: Gelechiidae). J. Econ. Entomol. 94: 1571-1576.

Carrière, Y., C. Ellers-Kirk, A. L. Patin, M. A. Sims, S. Meyer, Y. B. Liu, T. J. Dennehy, and B. E. Tabashnik. 2001b. Overwintering cost associated with resistance to trans- genic cotton in the pink bollworm (Lepidoptera: Gelechiidae). J. Econ. Entomol. 94: 935-941.

Carrière, Y., C. Ellers-Kirk, M. Sisterson, L. Antilla, M. Whitlow, T. J. Dennehy, and B. E. Tabashnik. 2003. Longterm regional suppression of pink bollworm by Bacillus thuringiensis cotton. Proc. Natl. Acad. Sci. U.S.A. 100: 1519-1523. (doi: 10.1073/pnas.0436708100).

Carrière, Y., C. Ellers-Kirk, R. Biggs, B. Degain, D. Holley, C. Yafuso, P. Evans, T. J. Dennehy, and B. E. Tabashnik. 2005a. Effects of cotton cultivar on fitness costs associated with resistance of pink bollworm (Lepidoptera: Gelechiidae) to Bt cotton. J. Econ. Entomol. 98: 947-954. (doi: 10.1002/ps.1039).

Carrière, Y., C. Ellers-Kirk, K. Kumar, S. Heuberger, M. Whitlow, L. Antilla, T. J. Dennehy, and B. E. Tabashnik. 2005b. Long-term evaluation of compliance with refuge requirements for Bt cotton. Pest Manag. Sci. 61: 327-330. (doi: 10.1002/ps. 1039).

Comins, H. N. 1977. The development of insecticide resistance in the presence of migration. J. Theor. Biol. 64: 177-197.

Dyck, V. A., J. Hendrichs, and A. S. Robinson [eds.]. 2005. Sterile insect technique: principles and practice in areawide integrated pest management. Springer, Amsterdam, The Netherlands.

[EPA] The Environmental Protection Agency. 1998. The Environmental Protection Agency's white paper on Bt plant-pesticide resistance management. Office of Pesticides Programs, U.S. Environmental Protection Agency, Washington, DC.

Frisvold, G. B., and J. M. Reeves. 2008. The costs and benefits of refuge requirements: the case of Bt cotton. Ecol. Econ. 65: 87-97. (doi:10.1016/j.ecolecon.2007.06.002).

Fu, G., K. C. Condon, M. J. Epton, P. Gong, L. Jin, G. C. Condon, N. I. Morrison, T. H. Dafa'Alla, and L. Alphey. 2007. Female-specific insect lethality engineered using alternative splicing. Nat. Biotechnol. 25: 353-357. (doi: $10.1038 /$ nbt1283).

Gould, F. 1998. Sustainability of transgenic insecticidal cultivars: integrating genetics and ecology. Annu. Rev. Entomol. 43: 701-726.

Gould, F., A. Anderson, A. Jones, D. Sumerford, D. G. Heckel, J. Lopez, S. Micinski, R. Leonard, and M. Laster. 1997. Initial frequency of alleles for resistance to Bacillus thuringiensis toxins in field populations of Heliothis virescens. Proc. Natl. Acad. Sci. U.S.A. 94: 3519-3523.

Gujar, G. T., V. Kalia, A. Kumari, B. P. Singh, A. Mittal, R. Nair, and M. Mohan. 2007. Helicoverpa armigera baseline susceptibility to Bacillus thuringiensis Cry toxins and resistance management for Bt cotton in India. J. Invertebr. Pathol. 95: 214-219.

Gutierrez, A. P., and S. Ponsard. 2006. Physiologically based demographics of Bt cotton-pest interactions: I. Pink bollworm resistance, refuge and risk. Ecol. Model. 191: 346.

Huang, J., R. Hu, C. Pray, F. Qiao and S. Rozelle. 2003. Biotechnology as an alternative to chemical pesticides: a case study of Bt cotton in China. Agric. Econ. 29: 55-67. (doi:10.1016/S0169-5150(03)00044-6).

Huang, F., B. R. Leonard, and D. A. Andow. 2007. Sugarcane borer (Lepidoptera: Crambidae) resistance to transgenic Bacillus thuringiensis maize. J. Econ. Entomol. 100: 164171.

James, C. 2007. Global status of commercialized biotech/GM crops: 2007 ISAAA Brief No. 37. International Service for the Acquisition of Agri-biotech Applications, Ithaca, NY.

Liu, F., Z. Xu, J. Chang, J. Chen, F. Meng, Y. C. Zhu, and J. Shen. 2008. Resistance Allele frequency to Bt cotton in 
field populations of Helicoverpa armigera (Lepidoptera: Noctuidae) in China. J. Econ. Entomol. 101: 933-943.

Liu, Y. B., B. E. Tabashnik, T. J. Dennehy, A. L. Patin, M. A. Sims, S. K. Meyer, and Y. Carrière. 2001. Effects of Bt cotton and CrylAc toxin on survival and development of pink bollworm (Lepidoptera: Gelechiidae). J. Econ. Entomol. 94: 1237-1242.

Luttrell, R. G., and M. I. Ali. 2007. Exploring selection for Bt resistance in heliothines: results of laboratory and field studies, pp. 1073-1086. In Proceedings, Beltwide Cotton Conference, New Orleans, LA.

Mahon, R. J., K. M. Olsen, S. Downes, and S. Addison. 2007. Frequency of alleles conferring resistance to the Bt toxins CrylAc and Cry2Ab in Australian populations of Helicoverpa armigera (Lepidoptera: Noctuidae). J. Econ. Entomol. 100: 1844-1853.

Marrelli, M. T., C. K. Moreira, D. Kelly, L. Alphey, and M. Jacobs-Lorena. 2006. Mosquito transgenesis: what is the fitness cost? TRENDS in Parasitology 22: 197-202. (doi: 10.1016/j.pt.2006.03.004).

Ramachandran, S., G. D. Buntin, J. N. All, B. E. Tabashnik, P. L. Raymer, M. J. Adang, D. A. Pulliam, and C. N. Stewart, Jr. 1998. Survival, development, and oviposition of resistant diamondback moth (Lepidoptera: Plutellidae) on transgenic canola producing a Bacillus thuringiensis toxin. J. Econ. Entomol. 91: 1239-1244.

Raymond, B., A. H. Sayyed, and D. J. Wright. 2005. Genes and environment interact to determine the fitness costs of resistance to Bacillus thuringiensis. Proc. R. Soc. B: Biol. Sci. 272: 1519-1524. (doi: 10.1098/rspb.2005.3103).

Reynolds, A. 2007. Why EPA thinks resistance monitoring for Bt crops has been important and where do we go from here, Entomological Society of America Annual Meeting, San Diego, CA. (http://esa.confex.com/esa/2007/techprogram/paper 27964.htm).

Shelton, A. M., J. Z. Zhao, and R. T. Roush. 2002. Economic, ecological, food safety, and social consequences of the deployment of Bt transgenic plants. Annu. Rev. Entomol. 47: 845-881.
Sisterson, M. S., L. Antilla, Y. Carrière, C. Ellers-Kirk, and B. E. Tabashnik. 2004. Effects of insect population size on evolution of resistance to transgenic crops. J. Econ. Entomol. 97: 1413-1424.

Stodola, T. J., D. A. Andow, A. R. Hyden, J. L. Hinton, J. J. Roark, L. L. Buschman, P. Porter, and G. B. Cronholm. 2006. Frequency of resistance to Bacillus thuringiensis toxin CrylAb in southern United States corn belt population of European corn borer (Lepidoptera: Crambidae). J. Econ. Entomol. 99: 502-507.

Tabashnik, B. E., A. L. Patin, T. J. Dennehy, Y. B. Liu, Y. Carrière, M. A. Sims, and L. Antilla. 2000. Frequency of resistance to Bacillus thuringiensis in field populations of pink bollworm. Proc. Natl. Acad. Sci. U.S.A. 97: 1298012984.

Tabashnik, B. E., T. J. Dennehy, and Y. Carrière. 2005. Delayed resistance to transgenic cotton in pink bollworm. Proc. Natl. Acad. Sci. U.S.A. 102: 15389-15393. (doi 10.1073/pnas.0507857102).

Tabashnik, B. E., A. J. Gassmann, D. W. Crowder, and Y. Carriere. 2008. Insect resistance to Bt crops: evidence versus theory. Nat Biotech 26: 199-202. (doi:10.1038/ nbt1382).

Tang, J. D., H. L. Collins, R. T. Roush, T. D. Metz, E. D. Earle, and A. M. Shelton. 1999. Survival, weight gain, and oviposition of resistant and susceptible Plutella xylostella (Lepidoptera: Plutellidae) on broccoli expressing Cry1Ac toxin of Bacillus thuringiensis. J. Econ. Entomol. 92: $47-55$.

Thomas, D. D., C. A. Donnelly, R. J. Wood, and L. S. Alphey. 2000. Insect population control using a dominant, repressible, lethal genetic system. Science (Wash., D.C.) 287: 2474-2476.

Wallace, B. 1968. Topics in population genetics. Norton, New York.

Received 17 July 2008; accepted 3 November 2008. 